OPEN ACCESS

Edited by:

Pablo Engel,

University of Barcelona, Spain

Reviewed by:

Benedict Seddon,

University College London,

United Kingdom

Koji Tokoyoda,

German Rheumatism Research

Center (DRFZ), Germany

*Correspondence:

Francesca Di Rosa francesca.dirosa@cnr.it

Specialty section:

This article was submitted to

Viral Immunology,

a section of the journal

Frontiers in Immunology

Received: 15 January 2021

Accepted: 15 March 2021

Published: 09 April 2021

Citation:

Di Rosa F, Cossarizza A and Hayday AC (2021) To Ki or Not to Ki: Re-Evaluating the Use and Potentials of Ki-67 for $T$ Cell Analysis.

Front. Immunol. 12:653974. doi: 10.3389/fimmu.2021.653974

\section{To Ki or Not to Ki: Re-Evaluating the Use and Potentials of Ki-67 for T Cell Analysis}

\author{
Francesca Di Rosa ${ }^{1 *}$, Andrea Cossarizza ${ }^{2,3}$ and Adrian C. Hayday ${ }^{4,5,6}$ \\ 1 Institute of Molecular Biology and Pathology, National Research Council of Italy (CNR), Rome, Italy, ${ }^{2}$ Department of Medical \\ and Surgical Sciences for Children and Adults, University of Modena and Reggio Emilia, Modena, Italy, ${ }^{3}$ National Institute for \\ Cardiovascular Research, Bologna, Italy, ${ }^{4}$ Immunosurveillance Laboratory, The Francis Crick Institute, London, United \\ Kingdom, 5 Peter Gorer Department of Immunobiology, King's College London, London, United Kingdom, 6 National Institute \\ for Health Research (NIHR) Biomedical Research Center (BRC), Guy's and St Thomas' NHS Foundation Trust and King's \\ College London, London, United Kingdom
}

This study discusses substantive advances in T cell proliferation analysis, with the aim to provoke a re-evaluation of the generally-held view that Ki-67 is a reliable proliferation marker per se, and to offer a more sensitive and effective method for $T$ cell cycle analysis, with informative examples in mouse and human settings. We summarize recent experimental work from our labs showing that, by Ki-67/DNA dual staining and refined flow cytometric methods, we were able to identify $T$ cells in the $S-G_{2} / M$ phases of the cell-cycle in the peripheral blood (collectively termed "T Double S" for I cells in S-phase in Sanguine: in short "TDS" cells). Without our refinement, such cells may be excluded from conventional lymphocyte analyses. Specifically, we analyzed clonal expansion of antigen-specific CD8 $T$ cells in vaccinated mice, and demonstrated the potential of $T_{D S}$ cells to reflect immune dynamics in human blood samples from healthy donors, and patients with type 1 diabetes, infectious mononucleosis, and COVID-19. The Ki-67/DNA dual staining, or $\mathrm{T}_{\mathrm{DS}}$ assay, provides a reliable approach by which human peripheral blood can be used to reflect the dynamics of human lymphocytes, rather than providing mere steady-state phenotypic snapshots. The method does not require highly sophisticated "-omics" capabilities, so it should be widely-applicable to health care in diverse settings. Furthermore, our results argue that the $T_{D S}$ assay can provide a window on immune dynamics in extra-lymphoid tissues, a long-sought potential of peripheral blood monitoring, for example in relation to organ-specific autoimmune diseases and infections, and cancer immunotherapy.

Keywords: flow cytometry, T cells, cell cycle, Ki-67, DNA dye

\section{INTRODUCTION}

Quantitation of Ki-67, a nuclear protein associated with cell cycle, is currently among the topranked methods to evaluate $\mathrm{T}$ cell proliferation, especially in human samples ex vivo. Readily detectable levels of Ki-67 mRNA and protein are present during the four cell cycle phases (i.e., $\mathrm{G}_{1}, \mathrm{~S}$, $\left.G_{2}, M\right)$ and are down-regulated when cells exit cell cycle and enter into quiescence (i.e., the $G_{0}$ phase) (1). Originally named after the first monoclonal antibody (mAb) used to identify it (2), Ki-67 
protein can now be stained with a series of mAbs with different sensitivities and epitope-specificities, including some mAbs that can detect extremely low levels of the protein, even in quiescent cells (3-6). From a functional standpoint, Ki-67 supports chromosome architecture organization and nucleolar assembly upon mitosis $(7,8)$; helps remove cytoplasm from the reassembling nucleus during mitotic exit (9); and regulates heterochromatin compaction and gene expression in proliferating cells (10). This notwithstanding, mutant mice with disrupted Ki-67 expression are vital and fertile, grow normally, and do not show abnormalities in highly proliferative tissues, such as the intestinal epithelium (10).

Given these considerations, it is evident that the very frequent use of Ki-67 as a proliferation marker is mistaken: rather, Ki-67 discriminates between cells having detectable Ki-67 expression (Ki$67^{+}$) in any phase of cell cycle (i.e., $G_{1}, S, G_{2}, M$ ), and cells lacking it (Ki-67) in the quiescent state $G_{0}$. Notably, the $G_{1}$ phase can be considered a cell cycle hub of highly variable duration. Thus, a Ki$67^{+}$cell in $G_{1}$ can derive either from cell cycle entry of a cell that was previously in $\mathrm{G}_{0}$, or a mitotic event that generates two daughter cells that sustain in $\mathrm{G}_{1}$. Indeed, an often-neglected notion is that the subsequent fate of a $\mathrm{Ki}-67^{+}$cell in $\mathrm{G}_{1}$ can be any one of the following: i) to remain in $G_{1}$ for a long time; ii) to rapidly proceed into $S-G_{2} / M$; iii) to move into $G_{0}$, going out of cell cycle.

Adding a layer of complexity, Ki-67 levels in $G_{1}$ and $G_{0}$ also depend on the time a cell has spent in that phase, as the protein is degraded continuously during $G_{1}$ and $G_{0}$, while it accumulates in $S, G_{2}, M(6)$. Some critical issues related to Ki-67 protein half-life emerged from a series of elegant studies addressing mouse B and $\mathrm{T}$ cell proliferation dynamics by in vivo experiments plus mathematical modeling (11-14). In some of these studies, Ki67 staining was used in combination with other methods, including mouse treatment with bromodeoxyuridine (BrdU), a thymidine analogue which is incorporated into DNA during Sphase, and adoptive transfer of $\mathrm{T}$ cells labeled with carboxyfluorescein succinimidyl ester (CFSE), a fluorescent dye that is equally distributed between daughter cells upon division $(11,12)$. It was observed that Ki-67 level of expression by Ki-67 ${ }^{+}$ cells may vary depending on cell division history. Thus, Ki-67 expression by CFSE-labeled T cells transferred in lymphopenic mice was extremely high in cells that had undergone many rounds of divisions, and low in recently divided cells whose proliferation was inhibited by adoptive transfer of large numbers of competing $\mathrm{T}$ cells (11). The possibility that Ki-67 expression could reflect a recent post-mitotic state was reinforced by BrdU pulse-chase data, showing that BrdU-labeled CD4 $\mathrm{T}_{\mathrm{EM}}$ and $\mathrm{T}_{\mathrm{CM}}$ cells were still Ki- $67^{+} 4$ days after BrdU-treatment withdrawal (12). Furthermore, it was highlighted that Ki-67 expression by a differentiated $\mathrm{T}$ or $\mathrm{B}$ cell could derive from Ki-67 protein inheritance following cell division at a previous developmental stage, rather than reflect proliferation of the Ki- $67^{+}$cell itself $(13$, 14). In short, evaluation of Ki-67 as a single marker to define a proliferative state incurs risk of misinterpretation.

In this article, we will discuss how these considerations are brought into focus by recent findings on proliferating $\mathrm{T}$ cells in the peripheral blood of healthy subjects and those with diseases
$(15,16)$, including COVID-19 (17). We will briefly describe how a highly sensitive and effective flow cytometric method based on Ki-67/DNA dual staining, the $\mathrm{T}_{\mathrm{DS}}$ assay, provided a practical and reliable approach to distinguish between $T$ cells in $G_{1}$ and those in $S-G_{2} / M$ phases of cell cycle (15-17). Finally, we will advocate incorporating the $\mathrm{T}_{\mathrm{DS}}$ assay into routine immuno-monitoring.

\section{KI-67/DNA DUAL STAINING OF T CELLS}

Refined kinetics studies on $\mathrm{T}$ cell clonal expansion after vaccination in a mouse model showed that antigen-specific CD8 $\mathrm{T}$ cells from blood contained on average 8-fold more cells in $S-G_{2} / M$ at day 3 versus day 7 post-boost, even though both the percentage of Ki- $67^{+}$cells and the antigen-specific CD8 T cell frequency were much higher at day 7 (Supplemental Table 1A, and (15)). These results suggested that the peak of actively cycling cells anticipates by a few days the peak of Ki- $67^{+}$cells at day 7 , which might reflect entry of cells into a prolonged $G_{1}$ phase after a recent mitosis and/or cell mobilization into the blood. By contrast, only a small proportion of total CD8 T cells from control untreated mice were in $G_{1}$, and very few were in S$\mathrm{G}_{2} / \mathrm{M}$ at any time point (Supplemental Table 1A).

In these experiments, the limitations of using Ki-67 as a single marker were overcome by a flow cytometric method based on dual staining of Ki-67 and DNA, that allows a clear distinction of cells in $\mathrm{G}_{0}(\mathrm{Ki67} / \mathrm{DNA} 2 \mathrm{n})$, from those in $\mathrm{G}_{1}\left(\mathrm{Ki}^{-} 7^{+} / \mathrm{DNA} 2 \mathrm{n}\right)$, and from those in $\mathrm{S}-\mathrm{G}_{2} / \mathrm{M}\left(\mathrm{Ki} 67^{+} / 2 \mathrm{n}<\mathrm{DNA} \leq 4 \mathrm{n}\right)$, as previously demonstrated by studies of bone marrow Hematopoietic Stem Cells (HSC) (18). When adapting this protocol to ex vivo analysis of antigen-specific T cells of mouse spleen, lymph nodes (LN) and blood, an unconventional strategy for data analysis was employed that included events with high Forward (FSC) and Side Scatter (SSC) (15). Such events are commonly discarded when examining lymphocytes in freshly obtained heterogeneous tissue samples, in order to exclude cell aggregates and myeloid cells that are typically more auto-fluorescent than lymphocytes.

In the mouse vaccination study, the unconventional combination of a DNA-Area/DNA-Width criterion, that is normally used in HSC cell cycle studies to exclude cell aggregates and debris (19), with a "relaxed" lymphocyte gate for FSC and SSC, allowed the ready detection of T cells in the S$\mathrm{G}_{2} / \mathrm{M}$ phases of the cell cycle that might have been missed with a standard gating strategy, and offered enhanced sensitivity in measuring cells in $G_{1}$ (15). Indeed, comparison of mouse LN data obtained applying either the conventional lymphocyte gate excluding cells with high FSC and SSC, or the "relaxed" gate that included cells with high FSC and SSC, revealed that at day 3 postboost the conventional gate under-estimated antigen-specific $\mathrm{CD} 8 \mathrm{~T}$ cell frequency and the $\mathrm{Ki}-67^{+}$proportion among antigen-specific CD8 $\mathrm{T}$ cells by an average of 6 -fold, and 3fold, respectively (15). These results suggest that the current criteria of analysis of ex vivo mouse samples are appropriate for resting $\mathrm{T}$ cells, but are not optimal for activated $\mathrm{T}$ cells, e.g. those cycling during early phases of an immune response to vaccination. 


\section{VALIDATION BY IMAGE FLOW CYTOMETRY}

The prospect that antigen-responding $\mathrm{T}$ cells proceeding into cell cycle share with blast cells traits including increasing size and modifications of internal organelles has been validated by image flow cytometry analysis of a TCR transgenic mouse CD8 T cell population following stimulation with its cognate antigen in vitro (16). In these studies, the combination of flow cytometry and microscopy permitted visualization and quantitative multiparameter characterization of $\mathrm{T}$ cells in different phases of the cell cycle, as identified by Ki-67/DNA dual staining: thus antigen-induced $\mathrm{T}$ cell cycle progression corresponded to quantitative increases in SSC, nuclear size (DNA area), cell size (brightfield area), and uptake of a mitochondrial marker (16).

Notably, T cells with such features of cycling blast cells were identified in the peripheral blood of some healthy donors (HD), without any in vitro stimulation (16). In this context, Ki-67 staining resolved an unexpected technical issue, i.e. the presence of a few cell aggregates (about $0.3 \%$ of the CD8 T cells) that could not be eliminated based on the DNA-only criterion and which appeared as Ki-67 events having 4n DNA content. Visualization of these events by image flow cytometry analysis showed that they were doublets formed by one cell sitting almost on top of another cell, thereby appearing like a shadow ("shadow" doublets) (16). This type of potential artifact involving only a tiny cell fraction had been previously reported, and solved by microscopy-based high content screening (20). The Ki-67/DNA dual staining offered the option to exclude "shadow" doublets by flow cytometry, i.e. by gating out Ki67/4n DNA events (16).

\section{THE TDS ASSAY FOR REFINED IMMUNO-MONITORING}

Following the technical resolution of "shadow" doublets, we could consistently apply flow cytometry to detect and quantitate in HD blood $T$ cells in the $S-G_{2} / M$ phases of cell cycle, collectively called "T Double S" for T cells in $\underline{\mathrm{S}}$-phase in Sanguine, in short $\mathrm{T}_{\mathrm{DS}}$ (16). Indeed, $\mathrm{T}_{\mathrm{DS}}$ were clearly evident in $\mathrm{T}_{\text {reg }}$ cells Fraction II (CD4 $4^{+}$FoxP3 ${ }^{\text {high }}$ CD45RA ${ }^{-} \mathrm{T}$ cells) from $\mathrm{HD}$, being on average $0.82 \%$, and suggesting ongoing immune regulation. $\mathrm{T}_{\mathrm{DS}}$ were rare among conventional $\mathrm{T}$ cell memory subsets, and more frequently represented in CD4 and CD8 $\mathrm{T}_{\mathrm{EM}}$ cells. In contrast, $\mathrm{T}_{\mathrm{DS}}$ were almost completely absent among $\gamma \delta \mathrm{T}$ cells (16). A typical example of flow cytometry data demonstrating $\mathrm{T}_{\mathrm{DS}}$ enrichment in CD8 $\mathrm{T}_{\mathrm{EM}}$ cells is represented in Figure 1, that shows general gating strategy (panel A), identification of naïve/memory CD8 $\mathrm{T}$ cell subsets (panel B), and cell cycle analysis for each subset (panel C).

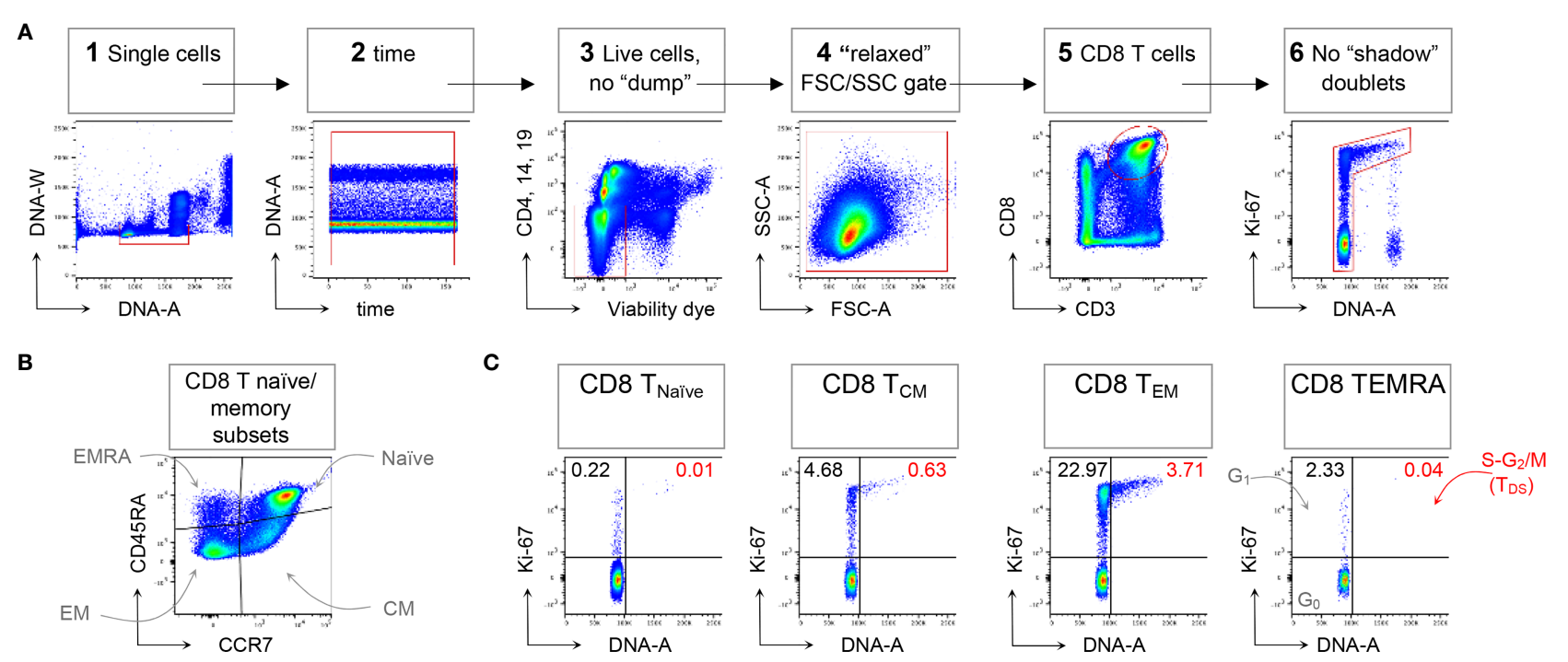

FIGURE 1 | Example of CD8 T cell naïve/memory subset analysis by $T_{\mathrm{DS}}$ assay. HD PBMCs were stained with the viability dye eFluor 780 (eF780), the DNA dye Hoechst-33342, and fluorochrome conjugated mAbs against surface markers and Ki-67, as described (16). An example of flow cytometry analysis is shown. (A) Gating of viable single CD8 T cells in 6 steps: 1) DNA-A-W singlets. Single cells having $2 n \leq$ DNA content $\leq 4 n$ were selected on the DNA-area (A) versus (vs) DNA-width (W) plot; 2) Time exclusion. Stable acquisition over time (seconds) was monitored on the time vs DNA-A plot and any events collected in case of pressure fluctuations were excluded; 3) Viable cells, no "dump". Cells expressing CD4, CD14 and CD19, and dead cells were excluded; 4) FSC-ASSC-A "relaxed" gate. A "relaxed" gate was used on the FSC-A vs SSC-A plot, to include highly activated and cycling lymphocytes (15); 5) CD8 T cells. CD8 T cells were gated on the CD3 versus CD8 plot; 6) Refined singlets. A few remaining doublets composed by one cell sitting on top of another (so-called shadow doublets) were excluded as Ki-67int/ events having $>2 \mathrm{n}$ DNA content (16). This gating strategy was used as a base for the subsequent gates. (B) The following naïve/memory subsets of CD8 T cells were identified: CD45RA ${ }^{+}$ $\mathrm{CCR}^{+}$Naïve, CD45RA- CCR7 ${ }^{+}$central memory (CM), CD45RA- CCR7 ${ }^{-}$effector memory (EM), and CD45RA ${ }^{+}$CCR7 ${ }^{-}$(EMRA). (C) Cell cycle phases of each naïve/ memory CD8 T cell subset were defined on DNA-A vs Ki67-A plot as follows: cells in $\mathrm{G}_{0}$ were identified as DNA 2n/Ki67- (bottom left quadrant); cells in $\mathrm{G}_{1}$ as DNA 2n/ $\mathrm{Ki}_{6} 7^{+}$(upper left quadrant); cells in $\mathrm{S}-\mathrm{G}_{2} / \mathrm{M}$ (or $\mathrm{T}_{\mathrm{DS}}$ cells) as $\mathrm{DNA}>2 \mathrm{n} / \mathrm{Ki} 67^{+}$(top right quadrant). Unpublished data in relation to (16). 
These results prompted further investigation in human diseases employing Ki-67/DNA dual staining and flow cytometric analysis: the $\mathrm{T}_{\mathrm{DS}}$ assay. In Infectious Mononucleosis (IM), the clinical manifestation of primary EBV infection, CD8 T cells specific for a single EBV immunodominant epitope contained up to $80 \%$ of cells in $\mathrm{G}_{1}$ and up to $20 \%$ of $\mathrm{T}_{\mathrm{DS}}$ cells, whereas corresponding cells in healthy EBV carriers contained about $5 \%$ of cells in $\mathrm{G}_{1}$ and $0 \% \mathrm{~T}_{\mathrm{DS}}$. In fact, $\mathrm{T}_{\mathrm{DS}}$ performed better than the frequency of EBV-specific CD $8 \mathrm{~T}$ cells for discriminating IM patients from healthy EBV carriers (16).

In Type 1 Diabetes (T1D), the $\mathrm{T}_{\mathrm{DS}}$ assay was applied to CD8 $\mathrm{T}$ cells specific for antigens of Islets of Langerhans, the target organ of the pathogenic autoimmune attack. An evident difference in $\mathrm{T}_{\mathrm{DS}}$ representation emerged between patients and $\mathrm{HD}$, that was quantitated as follows: considering $0.248 \% \mathrm{~T}_{\mathrm{DS}}$ (i.e. $\mathrm{HD}$ mean $+3 \mathrm{xSD})$ as a threshold, individuals with $\mathrm{T}_{\mathrm{DS}}$ percentage values above this $\left(T_{D S}^{+}\right)$comprised about half of the T1D cohort, and were absent among HD (16). The two subsets of T1D patients $-T_{D S}^{+}$and $T_{D S}^{-}-$showed significantly different $T_{D S}$ representation among islet-specific CD8 $\mathrm{T}$ cells but not among anti-viral CD8 T cells (16) or total CD8 T cells (Supplemental Table 1B). A prominent rise in islet-specific CD8 $\mathrm{T}_{\mathrm{DS}}$ cells $(>3 \%)$ was associated with an aggressive effector phenotype of the islet-specific CD8 T cells in the blood. Thus, $\mathrm{T}_{\mathrm{DS}}$ measurement may have immediate clinical utility offering extra insight into the progression of a disease which can be challenging to track by other means (16).

Finally, the $\mathrm{T}_{\mathrm{DS}}$ assay was used in an immune monitoring study ("COVID-IP") of hospital-treated COVID-19 patients (17). Among the key traits of a consensus COVID-19 immune signature identified by the study was a dysregulated $\mathrm{T}$ cell response characterized by concurrent cytopenia, activation, proliferation, and exhaustion. The $\mathrm{T}_{\mathrm{DS}}$ assay documented that patients with a severe disease progression had a higher percentage of cells in $\mathrm{G}_{1}$ and of $\mathrm{T}_{\mathrm{DS}}$ cells among CD4 and CD8 $\mathrm{T}_{\mathrm{EM}}$ cells, as compared with HD. $\gamma \delta \mathrm{T}$ cells in $\mathrm{G}_{1}$ were similarly increased but those cells were not associated with increases of cells in $S-G_{2} / M$. All such changes were less evident in patients with a moderate disease evolution, with a significant difference between the two patient groups (Supplemental Table 1C). Hence, in this setting too, the ready quantitation of $T$ cells in $G_{1}$ and $T_{D S}$ cells could contribute to patient discrimination. Lastly, blood $\mathrm{T}$ cell analysis by Ki-67/ DNA dual staining helped to identify critical immunological traits of COVID-19 patients with either solid or haematological cancers versustheir non-COVID counterparts, further confirming the great utility of the $\mathrm{T}_{\mathrm{DS}}$ assay in immunomonitoring (21).

\section{REFERENCES}

1. Scholzen T, Gerdes J. The Ki-67 protein: from the known and the unknown. J Cell Physiol (2000) 182:311-22. doi: 10.1002/(SICI)1097-4652(200003) 182:3<311::AID-JCP1>3.0.CO;2-9

2. Gerdes J, Lemke H, Baisch H, Wacker HH, Schwab U, Stein H. Cell cycle analysis of a cell proliferation-associated human nuclear antigen defined by the monoclonal antibody Ki-67. J Immunol (1984) 133:1710-5.

\section{CONCLUDING REMARKS}

In sum, the value of Ki-67 as an informative marker of cell cycle status can be greatly enhanced by its use in combination with a DNA stain, as described in the $\mathrm{T}_{\mathrm{DS}}$ assay. The routine flow cytometry application of this might usefully be used to better understand $\mathrm{T}$ cell biology, to monitor responses to vaccination and treatment, and to gain early warnings of spontaneous disease exacerbation or remission. The possibility of using more sophisticated approaches, based upon multilaser excitation of molecules such as CSFE for counting cell divisions, or fluorochrome-conjugated antibodies recognizing cyclins differentially expressed during the cell cycle (22) or even mass cytometry (23) may soon permit a better understanding of, and hence better deployment of, Ki-67 in tracking and studying cell cycling as a key component of immune regulation.

\section{AUTHOR CONTRIBUTIONS}

FD wrote the manuscript with help by $\mathrm{AH}$ and insightful inputs by AC. Experimental studies were performed in the labs of FD and $\mathrm{AH}$. All authors contributed to the article and approved the submitted version.

\section{FUNDING}

Funding for this study was provided by Royal Society grant IES $\backslash$ R3 $\backslash 170319$ (to AH and FD) and Italian Minister of Research and University (MIUR) grant PRIN 2017K55HLC (to FD).

\section{ACKNOWLEDGMENTS}

We thank Ambra Natalini and Sonia Simonetti (FD lab), Miguel Muñoz-Ruiz, Leticia Monin, and Adam G. Laing (AH lab), plus Irma Pujol-Autonell (King's College London) and Hefin Rhys (the Francis Crick Institute) for their collaboration on the $T_{D S}$ assay, and all members of the labs of FD and AH for discussion.

\section{SUPPLEMENTARY MATERIAL}

The Supplementary Material for this article can be found online at: https://www.frontiersin.org/articles/10.3389/fimmu.2021. 653974/full\#supplementary-material

3. Bullwinkel J, Baron-Luhr B, Ludemann A, Wohlenberg C, Gerdes J, Scholzen T. $\mathrm{Ki}-67$ protein is associated with ribosomal RNA transcription in quiescent and proliferating cells. J Cell Physiol (2006) 206:624-35. doi: 10.1002/jcp.20494

4. Lindboe CF, Torp SH. Comparison of Ki-67 equivalent antibodies. J Clin Pathol (2002) 55:467-71. doi: 10.1136/jcp.55.6.467

5. Sun Y, Yang K, Bridal T, Ehrhardt AG. Robust Ki67 detection in human blood by flow cytometry for clinical studies. Bioanalysis (2016) 8:2399-413. doi: 10.4155/bio-2016-0194 
6. Miller I, Min M, Yang C, Tian C, Gookin S, Carter D, et al. Ki67 is a Graded Rather than a Binary Marker of Proliferation versus Quiescence. Cell Rep (2018) 24:1105-12.e5. doi: 10.1016/j.celrep.2018.06.110

7. Booth DG, Takagi M, Sanchez-Pulido L, Petfalski E, Vargiu G, Samejima K, et al. Ki-67 is a PP1-interacting protein that organises the mitotic chromosome periphery. Elife (2014) 3:e01641. doi: 10.7554/eLife.01641

8. Takagi M, Natsume T, Kanemaki MT, Imamoto N. Perichromosomal protein Ki67 supports mitotic chromosome architecture. Genes Cells (2016) 21:111324. doi: $10.1111 /$ gtc. 12420

9. Cuylen-Haering S, Petrovic M, Hernandez-Armendariz A, Schneider MWG, Samwer M, Blaukopf C, et al. Chromosome clustering by Ki-67 excludes cytoplasm during nuclear assembly. Nature (2020) 587:285-90. doi: 10.1038/ s41586-020-2672-3

10. Sobecki M, Mrouj K, Camasses A, Parisis N, Nicolas E, Lleres D, et al. The cell proliferation antigen Ki-67 organises heterochromatin. Elife (2016) 5:e13722. doi: $10.7554 /$ eLife.13722

11. Hogan T, Shuvaev A, Commenges D, Yates A, Callard R, Thiebaut R, et al. Clonally diverse $\mathrm{T}$ cell homeostasis is maintained by a common program of cell-cycle control. J Immunol (2013) 190:3985-93. doi: 10.4049/ jimmunol.1203213

12. Gossel G, Hogan T, Cownden D, Seddon B, Yates AJ. Memory CD4 T cell subsets are kinetically heterogeneous and replenished from naive $\mathrm{T}$ cells at high levels. Elife (2017) 6:1-30. doi: 10.7554/eLife.23013

13. Hogan T, Nowicka M, Cownden D, Pearson CF, Yates AJ, Seddon B, et al. Differential impact of self and environmental antigens on the ontogeny and maintenance of CD4 ${ }^{+}$T cell memory. Elife (2019) 8:1-23. doi: 10.7554/eLife.48901

14. Verheijen M, Rane S, Pearson C, Yates AJ, Seddon B. Fate Mapping Quantifies the Dynamics of B Cell Development and Activation throughout Life. Cell Rep (2020) 33:108376. doi: 10.1016/j.celrep.2020.108376

15. Simonetti S, Natalini A, Folgori A, Capone S, Nicosia A, Santoni A, et al. Antigen-specific CD8 $\mathrm{T}$ cells in cell cycle circulate in the blood after vaccination. Scand J Immunol (2019) 89:e12735. doi: 10.1111/sji.12735

16. Muñoz-Ruiz M, Pujol-Autonell I, Rhys H, Long HM, Greco M, Peakman M, et al. Tracking immunodynamics by identification of $S-G_{2} / M-$ phase $T$ cells in human peripheral blood. J Autoimmun (2020) 112:102466. doi: 10.1016/ j.jaut.2020.102466
17. Laing AG, Lorenc A, Del Molino Del Barrio I, Das A, Fish M, Monin L, et al. A dynamic COVID-19 immune signature includes associations with poor prognosis. Nat Med (2020) 26:1623-35. doi: 10.1038/s41591-020-1038-6

18. Wilson A, Murphy MJ, Oskarsson T, Kaloulis K, Bettess MD, Oser GM, et al. c-Myc controls the balance between hematopoietic stem cell self-renewal and differentiation. Genes Dev (2004) 18:2747-63. doi: 10.1101/gad.313104

19. Szade K, Bukowska-Strakova K, Zukowska M, Jozkowicz A, Dulak J. Analysis of Cell Cycle Status of Murine Hematopoietic Stem Cells. Methods Mol Biol (2016) 1516:91-9. doi: 10.1007/7651_2016_361

20. Kudernatsch RF, Letsch A, Stachelscheid H, Volk HD, Scheibenbogen C. Doublets pretending to be CD34+ T cells despite doublet exclusion. Cytometry A (2013) 83:173-6. doi: 10.1002/cyto.a.22247

21. Abdul-Jawad S, Baù L, Alaguthurai T, Del Molino Del Barrio I, Laing AG, Hayday TS, et al. Acute immune signatures and their legacies in severe acute respiratory syndrome coronavirus- 2 infected cancer patients. Cancer Cell (2021) 39:257-275. doi: 10.1016/j.ccell.2021.01.001

22. Martínez-Alonso D, Malumbres M. Mammalian cell cycle cyclins. Semin Cell Dev Biol (2020) 107:28-35. doi: 10.1016/j.semcdb.2020.03.009

23. Li N, van Unen V, Abdelaal T, Guo N, Kasatskaya SA, Ladell K, et al. Memory CD4+ T cells are generated in the human fetal intestine. Nat Immunol (2019) 20:301-12. doi: 10.1038/s41590-018-0294-9

Conflict of Interest: $\mathrm{AH}$ is a board member and equity holder in ImmunoQure, AG., and Gamma Delta Therapeutics, and is an equity holder in Adaptate Biotherapeutics.

The remaining authors declare that the research was conducted in the absence of any commercial or financial relationships that could be construed as a potential conflict of interest.

Copyright (c) 2021 Di Rosa, Cossarizza and Hayday. This is an open-access article distributed under the terms of the Creative Commons Attribution License (CC BY). The use, distribution or reproduction in other forums is permitted, provided the original author(s) and the copyright owner(s) are credited and that the original publication in this journal is cited, in accordance with accepted academic practice. No use, distribution or reproduction is permitted which does not comply with these terms. 